\section{INCUBACIÓN EMPRESARIAL EN LA UNIVERSIDAD NACIONAL MAYOR DE SAN MARCOS: UNA ESTRATEGIA PARA EFECTIVIZAR SU COMPROMISO CON EL DESARROLLO ECONÓMICO Y SOCIAL DEL PAÍS}

\author{
BUSINESS INCUBATION IN THE NATIONAL UNIVERSITY OF SAN \\ MARCOS: A STRATEGY FOR EFFECTUATE ITS COMMITMENT TO \\ ECONOMIC AND SOCIAL DEVELOPMENT OF THE COUNTRY
}

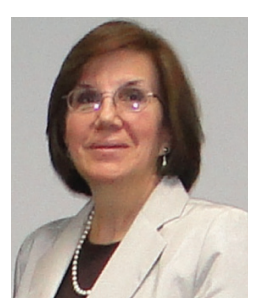

\author{
Ana María GutiérRez HubY* \\ Docente Principal de la Facultad de Ciencias Contables de la UNMSM
}

Colaboradora: Elsa Violeta Rafael Díaz

[Recepción: Julio de 2012/ Conformidad: Agosto de 2012]

\section{RESUMEN}

El presente artículo trata sobre la temática de la incubación empresarial universitaria buscando generar una corriente de opinión en autoridades, docentes y estudiantes de la Facultad de Ciencias Contables en torno a la importancia y necesidad de gestionar el desarrollo del potencial emprendedor del alumnado de la carrera contable, proyectándolo como una alternativa de acción que permita: canalizar una vinculación más efectiva entre la universidad y el medio empresarial, a la vez de concebirla como una de las estrategias para cumplir con la visión institucional de compromiso con el desarrollo económico y social del país.

Entre los aspectos expuestos se consideraron: la conceptualización de la incubación de empresas, su evolución y grado de desarrollo alcanzado, características e implicancias de los procesos de incubación, además de una revisión del contexto país y condiciones en el escenario socioeconómico y académico para la gestación de un programa de incubación empresarial.

A modo genérico se concluye que a nivel país es actualmente escaso el número de universidades que cuentan con programas de incubación y las pocas existentes todavía mantienen un escaso nivel de difusión en cuanto a su grado

\begin{abstract}
This study is about the enterprising incubation in universities that look for opinions from authorities, professors, and students in the Accounting Department in regards to the importance and necessity of the development of students who have entrepreneurial potential in the Accounting Department, helping the student with an alternative action that allows them to: canalize the most effective incaution between the university and the private sector, while at the same time to conceive it as one of the strategies that allows us to accomplish the institutional vision and the compromise with the social and economical development of the country.

Among the exposed aspects considered are: the conceptualization of the company incubation, its evolution and level of development, characteristics and implications of the incubation process, moreover a revision of a country context and the social, economic, and academic conditions of the private sector program.

We concluded that there are not enough universities that have an incubation program for entrepreneurial students, and the few that exist have to improve on their programs, Brazil and Mexico are the few countries that have these programs more so than any other country in
\end{abstract}

* Magister en Gestión Empresarial- UNMSM, Licenciada en Ciencias Administrativas. Docente investigadora de la Facultad de Ciencias Contables- UNMSM. E-mail: anamaria01053@yahoo.es 
de desarrollo; Brasil y México son los países latinoamericanos que mayor número de centros de incubación concentran; no obstante a nivel de Latinoamérica en términos generales, salvo escasas excepciones, la incubación empresarial todavía es un tema que se está forjando teniendo aun que superar limitaciones sobre todo en el tema de gestión e insuficiencia de recursos.

Palabras Clave: Incubación empresarial, emprendedurismo

\section{INTRODUCCIÓN}

Está ampliamente difundido que actualmente es latente el desafío de atender las nuevas temáticas provenientes de los países del primer mundo, tales como: sociedad de la información, economía del conocimiento, revolución digital, tecnología de la información y comunicación, entre otros, presentes en el medio gracias a la influencia de la globalización.

Es todo un proceso que, aunque recientemente se ha iniciado en el Perú, viene exigiendo respuestas inmediatas del Estado relacionadas con la generación de propuestas innovadoras, cambio de esquemas que solventen el desarrollo como país, mayor participación e integración de diversos agentes y sobre todo imposición de orden mediante estrategias concordadas que en conjunto impactan transversalmente en las actividades primordiales ligadas con el avance y desarrollo sostenible del país, como: la educación, la salud, la competitividad empresarial, las políticas sociales, la modernización del Estado, entre otros.

En este contexto, la educación superior, en especial las universidades por su naturaleza de ser instituciones encargadas de producir el conocimiento científico y tecnológico -elementos clave reconocidos en el progreso económico-, son las idóneas para asumir el compromiso de atender la demanda de the Latin American region. Entrepreneurial incubation is still a theme that is developing and it has to overcome challenges and limitations especially when it comes to lack of resources and insufficient.

Keywords: effective Incubation. Entrepreneurial potential. Effective incaution. Social and economic context.

contribuir ese desarrollo económico y social, como parte de su función misional y, por tanto, a una reflexión crítica sobre su vigencia y pertinencia frente a este escenario actual le sigue la búsqueda de estrategias para definir y operar los cambios necesarios internos que le permitan responder con eficacia a los procesos sociales de innovación demandados por el país, el cual a su vez le supone nuevas oportunidades para su propio desarrollo institucional.

Entre las estrategias para cumplir con su cometido se considera la asimilación del tránsito hacia un nuevo tipo de universidad a la que hacen referencia especialistas y estudiosos: una universidad que emprende, lo cual implica mucho más que apoyar la creación de empresas, es constituirse en una organización que se vincula y que interactúa abiertamente con su entorno social y económico adaptándose a los cambios y buscando recursos adicionales de financiación para sus actividades de investigación y enseñanza.

En efecto, en esta nueva visión de universidad, consciente de su importante rol en el proceso social de innovación, la vinculación con el entorno es crucial y refiere la conformación de una red de trabajo con el gobierno y con la empresa poniendo en marcha una dinámica de interacción en una tríada: universidad-empresa-Estado que conjugue entre sí en un marco común de cooperación y búsqueda del verdadero avance y desarro- 
1lo, lo que a la vez propiciará la generación de espacios para efectivizar la promoción de la innovación, considerando a los emprendedores como sus agentes centrales, a través de estrategias como la incubación empresarial universitaria, considerada una de las iniciativas organizacionales que se orientan precisamente a estimular la creación de actividades productivas y de innovación.

En consecuencia el objetivo del presente artículo es centrar la atención en el tema de la gestión del emprendedurismo estudiantil a través de la incubación empresarial universitaria en la perspectiva de ahondar en su dinámica, elementos que se conjugan e implicancias que podría suponer su aplicabilidad.

\section{QUÉ ES UNA INCUBADORA DE EM- PRESAS}

La incubación de empresas constituye "un proceso de soporte empresarial que acelera el desarrollo exitoso de empresas innovadoras proveyéndoles de un conjunto de recursos y servicios enfocados" (National Business Incubation Association, 2008).

En este sentido, una incubadora de empresa es:

"Un ambiente controlado que nutre la creación, el crecimiento y la protección de una nueva empresa, en una etapa temprana, antes de que ésta se encuentre lista para operar de manera autosostenible por sus propios medios. El término ambiente controlado abarca tanto elementos físicos, como virtuales" (Chinsomboon, 2000).

Cabe referir que en las definiciones encontradas sobre "incubadora de empresa" se advierte su construcción según los distintos puntos de vista de consultores y promotores, por lo que la definición mencionada es las más pertinente a juicio de la autora.

Así mismo cabe referir que los centros de incubación son operados: por instituciones educativas, organizaciones de la sociedad civil, organismos empresariales y asociaciones civiles, las cuales cuentan con un Modelo de Incubación reconocido.

\section{ORGANISMOS A NIVEL MUNDIAL QUE CONGREGAN INCUBADORAS}

Entre las asociaciones de centros de incubación más importantes se distinguen:

National Business Incubator Association (NBIA). Creada en 1987 por líderes de la industria norteamericana, es considerada la más avanzada mundialmente en incubación de empresas y fomento del emprendedurismo. Actualmente congrega más de 700 miembros y brinda información, capacitación, asesoría a miles de profesionales. ${ }^{1}$

European Business and Innovation Centres Network (EBN). Fundada en 1984, esta red europea tiene por objetivo promover el crecimiento de centros de innovación y negocios, congregando actualmente a más de 200 centros de incubación, emprendimiento e innovación. ${ }^{2}$

Japan Association of New Business Incubation Organizations (JANBO). Creada en 1999. Es la primera red de incubación en Japón, tiene desarrollado un sistema para la creación de empresas implementado en 47 prefecturas y 11 ciudades importantes del Japón además de soporte central.

Business Innovation and Incubation of Australia (BIIA). Establecida en 1995. Asociación de incubadoras de empresas que opera en Australia. Tiene más de 80 miembros. $^{3}$

\footnotetext{
1 http://www.nbia.org/

2 http://www.ebn.be/

3 http://www.revistasusp.sibi.usp.br/scielo.php?pid=S0080-21072006000400006\&script=sci_arttext
} 
Associação Nacional de Entidades Promotoras de Emprendimientos de Tecnologías Avançadas (ANPROTEC). Establecida en Brasil en 1987, está dedicada a crear mecanismos de apoyo a incubadoras de empresas, parques, polos tecnológicos, y otras entidades promotoras del emprendedurismo, cuenta con 330 asociados. ${ }^{4}$

\section{SURGIMIENTO Y EVOLUCIÓN DE LA INCUBACIÓN EMPRESARIAL}

Según la indagación, en cuanto a antigüedad e identificación de la primera incubadora de negocios no hay un consenso claro entre los autores, en lo que sí se coincide es en el reconocimiento que la incubación empresarial es iniciada en los Estados Unidos.

Se identificó tres versiones de surgimiento de la incubación: unos mencionan que el inicio de la práctica de la incubación de negocios se registra con el funcionamiento de la primera incubadora que surge en el año 1942 inicialmente con el propósito de incubar iniciativas estudiantiles por parte de la organización Student Agencies Inc. en Ithaca, New York ${ }^{5}$; también se alude a 1946 cuando empieza a funcionar la incubadora American Research Development (ARD) (Investigaciones para el Desarrollo de América) en el Massachusetts Institute of Technology, organización creada fuera del espacio universitario con el objetivo de proveer capital a las nuevas empresas de New England; también se menciona que fue a fines de 1959 en Batavia, New York, que aconteció el primer caso de incubadora denominada Batavia Industrial Center por la familia Mancuso, la cual es referida mayormente por los autores, lo cual se produce cuando el alcalde de Watertow,
Frank Mancuso, compró y adaptó una edificación para proveer de espacio a empresas de reciente creación donde antes funcionaba una incubadora de pollos que cerró dejando 2000 puestos de trabajo, a partir de allí se adoptaría y esparciría la idea de incubadora en otras ciudades de Estados Unidos, la incubadora recibió la denominación Mancuso Business Development Group propiciando la creación de más de 1200 empresas. ${ }^{6}$ El movimiento crecería lentamente las siguientes dos décadas.

Cabe mencionar que se advierten tres movimientos propulsores de la iniciativa de incubación, los que se forjan paralelamente en la década de los 50: el primero buscó encontrar nuevo uso para fábricas abandonadas en el medio oeste y noreste con el funcionamiento de pequeñas empresas; el segundo surgió como un experimento de la National Science Foundation para fomentar el emprendimiento e innovación en las universidades; y el tercero por iniciativa de emprendedores exitosos y grupos inversionistas que buscaban transferir sus experiencias a nuevas empresas en un entorno orientado al logro del éxito en la innovación tecnológica.

En los años 70 a raíz de las altas tasas de desempleo a causa del colapso de la industria tradicional, se reconoce en Estados Unidos y Europa la necesidad de formular estrategias para apoyar la recuperación de sectores, regiones y comunidades en estado crítico, siendo en esta década que se esparce el concepto de proveer el servicio asistencial a empresas en instalaciones compartidas. En este contexto se crean varias incubadoras de empresas, mayormente sin fines de lucro y en asociación con comunidades locales. Un considerable número de estas incubadoras

\footnotetext{
4 http://www.revistasusp.sibi.usp.br/scielo.php?pid=S0080-21072006000400006\&script=sci_arttext

5 Vela Velásquez, Julio César. (2011) “Modelo para la creación de incubadoras de empresas en la realidad peruana”. Tesis.

6 Awad Aubat, Gabriel. Diseño de un modelo de incubación de negocios utilizando herramientas de dinámica de sistemas. Tesis
}

134/ QUIPURAMAYoc | Vol. 20(37) 2012 
pertenecieron a universidades, parques de ciencia y centros de investigación, siendo organizadas con el fin de comercializar tecnología y, en el caso de las universidades el interés de dotar a sus estudiantes de experiencia en el mundo real.

Es así que en los años 80 operaban aproximadamente 12 incubadoras de negocio en EE.UU, consolidándose su percepción como herramienta popular del desarrollo económico, llegándoseles a considerar instrumentos de aceleración para la formación de empresas, además de soportes de la innovación y transferencia de tecnología.

A partir de entonces fue acelerado su incremento superándose la limitación del enfoque de desarrollo económico de la época en cuanto a considerar solamente la atracción de la industria y expansión de grandes corporaciones, por la perspectiva de desarrollar empresas para el sostenimiento de las economías locales, siendo las incubadoras de empresa una alternativa para este apoyo.

En cuanto a la evolución de las incubadoras se presentan tres generaciones de modelo de incubación yuxtapuestos a su resultado exitoso $^{7}$ :

En una primera generación, concurren dos momentos: primero las incubadoras sólo brindaban infraestructura a las empresas, posteriormente se incorporan servicios complementarios: logística, administración para negocios, asistencia y transferencia de tecnología, entre otros.

En una segunda generación los modelos de incubación se orientan a los diferentes sectores en que brindan atención: sector tecnológico, económico, así como también al tipo de propósito que orienta su gestión: como por ejemplo la creación de nuevas empresas, nuevas líneas de negocio, modernización e innovación, etc.

En la tercera y última generación las incubadoras definen su actuación en base al reconocimiento amplio de lo que es innovación -más allá del ámbito industrial incluyendo desde las no tecnológicas, las ecológicas, hasta sectores específicos como grupos vulnerables y las relacionadas con la innovación tecnológica.

\section{LA PRÁCTICA DE LA INCUBACIÓN EMPRESARIAL EN PERÚ}

En el Perú, la primera referencia de incubadora de empresa fue de iniciativa privada y data de hace 21 años. Corresponde al Centro de Transferencia Tecnológica a Universitarios San José-CTTU creado en 1991 en la Libertad como un proyecto de incubación orientado al ámbito de la agricultura con jóvenes de formación superior, como resultado de un estudio de la Consultora Recursos S.A. Resultó una experiencia exitosa que emprendió el objetivo de formar jóvenes empresarios, líderes y responsables. Actualmente esta iniciativa privada opera diversas modalidades de formación e incubación. Al término del programa el participante tiene potenciado sus capacidades de identificar y gestionar agronegocios, insertar su producción en el mercado y manejar tecnologías apropiadas. ${ }^{8}$

En la actualidad las incubadoras de empresas son especialmente de base tecnológica y en este caso los primeros esfuerzos institucionales son los del Centro de Innovación y Desarrollo de la Pontificia Universidad Católica del Perú (CIDE-PUCP) que

7 Según Lalkaka (2005), citado por Pilar Pérez Hernán y Alejandro Márquez Estrada en ponencia expuesta en el I Congreso Iberoamericano de Ciencia, Tecnología, Sociedad e Innovación (2006)

8 Informe del proyecto (2005) "Facilitación de financiamiento para negocios basados en la biodiversidad y apoyo a las actividades de desarrollo de mercado en la región andina" CONAM-CAF-FEF. 
opera desde 1995, el del Instituto de Investigación y Capacitación en Telecomunicaciones (INICTEL) desde el año 2000 y el de la Universidad de Piura, que funciona desde el año 2001.

En el caso de universidades, son limitados los recursos dispuestos para el sostenimiento de las incubadoras las que van de acuerdo a las posibilidades económicas de estas instituciones. El apoyo suele darse a través de asignación de espacios y equipos de gestión, los que muchas veces son compartidos con otras actividades académicas.

El estado por su parte apoya, a través del CONCYTEC con su programa INCUBACYT con una línea de promoción para incubadoras de base tecnológica, el cual articula con PERÚINCUBA, la Asociación Peruana de Incubadora de Empresas.

A pesar del apoyo, los recursos públicos destinados a la incubación aún son limitados en el contexto de la prioridad que debiera tener el desarrollo de la ciencia y la tecnología, observándose que el financiamiento de las incubadoras proviene en la casi generalidad de casos tanto de las propias instituciones que las mantienen, como de entidades privadas y de cooperación internacional. En este punto, un aspecto favorable que se presenta para el financiamiento de empresas y emprendedores son las entidades proveedoras de capital de riesgo. En términos de capital semilla, es casi generalidad que éste es provisto por los propios emprendedores. Cabe advertir que en Perú la gran oferta de fondos para microcréditos existente representa una oportunidad para ser aprovechada para el caso de emprendimientos tecnológicos.

\section{PERSPECTIVAS A NIVEL PAÍS RES- PECTO A LA INCUBACIÓN}

La incubación empresarial en el Perú se encuentra actualmente en un contexto mucho más propicio que en años pasados. Es así que recapitulando lo avanzado se distinguen perspectivas para el desarrollo del movimiento de incubación:

Económicamente los últimos años han sido estables para el país, lo que se traduce : en el crecimiento del producto bruto interno, una política activa de inserción en espacios internacionales como negociaciones de Tratados de Libre Comercio y apertura a la competencia internacional, además de la aparición en escena de sectores que presentan dinamismo como la industria de confecciones, la exportación de productos agrícolas tradicionales y no tradicionales y la minería que tradicionalmente es el sector generador de mayores divisas. Así mismo, la existencia de una conciencia creciente del rol de las exportaciones en el desarrollo del país. En este contexto, la integración de las incubadoras peruanas a las redes latinoamericanas homólogas es compatible con las políticas de integración desarrolladas por el Estado peruano en los últimos años. ${ }^{9}$

Tecnológicamente, se perciben oportunidades de desarrollo tecnológico y creación de empresa gracias a la Internet, así mismo, a pesar de la ausencia de políticas y recursos efectivos de promoción del sector tecnológico hay actualmente una base importante de empresas nacionales desarrolladoras de software, lo que indica el gran dinamismo del sector.${ }^{10} \mathrm{El}$ sector telecomunicaciones se ha

9 Facilitación de financiamiento para negocios basados en la biodiversidad y apoyo a las actividades de desarrollo de mercado en la región andina.

10 De acuerdo a APESOFT (Asociación Peruana de Empresas de Software). 
dinamizado, con la intervención de grandes empresas, un aumento considerable de uso de telefonía en la población, principalmente urbana, a ello se suma las grandes empresas que ofertan servicios y productos de tecnologías de información y un crecimiento de la oferta de capacitación relacionados con el mismo. ${ }^{11}$

Actualmente tanto las entidades públicas como las privadas reconocen que la micro, pequeña y mediana empresa en el Perú sustentan gran parte de la empleabilidad, por tanto diversas ONGs, instituciones educativas y entidades del Estado están desarrollando actividades de toda índole dedicadas a este sector. Algunas de estas instituciones vinculadas a la incubación de empresas comienzan a ser vistas como instrumentos de generación de nuevas empresas y también de un nuevo tejido productivo vinculado a la tecnología.

Un aspecto percibido como favorable al desarrollo de las incubadoras empresariales es el gran auge de la industria del microcrédito que atiende financieramente a la micro y pequeña empresa. Siendo necesario el establecimiento de mecanismos adecuados para emplear este tipo de financiamiento en las empresas de las incubadoras, en particular las tecnológicas.

En relación al apoyo de las instituciones del gobierno, cabe mencionar que en el Ministerio de Trabajo y Promoción del Empleo las políticas públicas dirigidas a la pequeña y mediana empresa (PYME), significaron un cambio positivo en las políticas de Estado promotoras del desarrollo de este sector. Otras entidades como el Ministerio de la Producción, el CONCYTEC y PROM-
PYME también desarrollan actividades para este sector. CONCYTEC, particularmente, considera financiamiento para la innovación como una de sus líneas de apoyo. En este sentido, se observa una convergencia de entidades del Estado en sus políticas públicas, lo cual es favorable para el desarrollo de las incubadoras de empresas.

En el contexto referido se advierte que en los últimos años se ha producido un cambio en las condiciones del entorno para el desarrollo de incubadoras empresariales, al existir en la actualidad mejores perspectivas que en el pasado. De ese modo, se ha ido avanzando de manera gradual en la formación de redes de incubadoras, avance que se ha conseguido con limitado apoyo público, no obstante hay un discurso favorable por parte de las entidades del Estado peruano para el desarrollo de estas iniciativas. Por tanto está en agenda la política pública y el apoyo efectivo a la incubación de empresas. Por lo pronto la independencia en cuanto al financiamiento público puede resultar positivo para el movimiento de incubación, pues propicia la búsqueda de soluciones autosostenibles para las incubadoras peruanas.

A lo expresado cabe señalar que la vinculación de los centros de incubación peruanos con redes similares de otros países latinoamericanos es un elemento favorable para dinamizar el movimiento a nivel nacional. Esta integración a nivel regional propiciará la creación de nuevos tejidos empresariales de cooperación y de alianzas estratégicas entre empresas tecnológicas para hacer frente en mejores condiciones a la competencia empresarial fuera de Latinoamérica.

11 Vela Velásquez, Julio César. Tesis: Modelo para la creación de incubadoras de empresas en la realidad peruana. 2011. 


\section{MARCO JURÍDICO PARA LA PRO- MOCIÓN DE LA INCUBACIÓN}

Respecto a políticas públicas en torno a la incubación empresarial, en el Perú sólo se han encontrado tres leyes relacionadas a la promoción de la pequeña empresa, de la tecnología y la innovación, entre ellas:

La Ley No 28015 (03.07.2003): Promociona y formaliza la micro y pequeña empresa, categoriza a las empresas incubadas como micro y pequeña empresa (Mype). En su artículo $25^{\circ}$ señala que: "El estado a través del Consejo Nacional de Ciencia y Tecnología (CONCYTEC) promueve, articula y operativiza la investigación e innovación tecnológica entre las universidades y centros de investigación con la MYPE"; así mismo su artículo $27^{\circ}$ menciona que: "El Estado promueve la oferta de servicios tecnológicos a las MYPE, facilitándoles acceso a financiamiento y centros de innovación tecnológica o de desarrollo empresarial".

Ley No 28303 (23.07.2004): A través de esta ley, en el artículo $31^{\circ}$ se refiere que "el Estado, a través del CONCYTEC en colaboración con gobiernos regionales, universidades y empresas privadas, fomenta la creación de parques tecnológicos. Otorgó un plazo de 180 días naturales al CONCYTEC para la elaboración del primer proyecto piloto de parques tecnológicos".

Ley No 28304 (30.07.2004): Ley de promoción del desarrollo económico y productivo. En su artículo $26^{\circ}$ detalla los elementos de un programa de desarrollo de capacidades empresariales municipales, incluyendo identificación de ventaja competitiva, banco nacional de perfiles de negocios, módulos de asesoría de creación de empresas, sistema de asistencia dirigida por empresarios líderes, entre otros.

\section{LA UNIVERSIDAD, LA ORGANIZA- CIÓN IDÓNEA PARA FOMENTAR EM- PRENDEDURISMO Y CREAR UNA IN- CUBADORA EMPRESARIAL}

En el marco de la denominada nueva economía, la conjugación entre la producción (empresa) y el conocimiento (universidad) es el factor clave para el crecimiento económico, apareciendo en escena la "innovación" como un elemento resultante de la interacción que pueda darse de esta alianza entre ambos factores.

En este sentido el papel de la universidad como espacio generador, transmisor y difusor del conocimiento, queda revalorizado a través de este reconocimiento a la importancia del conocimiento que mediante la investigación ella provee y que por tanto la posiciona como ente central del proceso social de innovación y desarrollo del país.

No obstante tal posición le implica a una universidad que ha transitado al modelo de universidad emprendedora, al desarrollar, mantener, consolidar e incrementar lazos de vinculación con las empresas en general; ello es una condición insoslayable para hacer efectiva la apertura de estrategias y espacios para la promoción de la innovación.

Al respecto, los espacios para promover la innovación, la formación en capacidades emprendedoras y el incentivo al emprendimiento a través de la implementación de centros de incubación empresarial al interior de los centros universitarios para la puesta en práctica de dichas capacidades, se presenta como una estrategia relevante para la concreción del referido propósito. ${ }^{12}$

12 Delicio, Fabián Andrés. Estrategias para el fomento del emprendedurismo en la Facultad de Ciencias Económicas y Sociales de la Universidad Nacional del Mar del Plata. Año 12, № 26, 2006. 
LA INCORPORACIÓN DEL EMPRENDEDURISMO EN EL PROCESO FORMATIVO: UNA ACCIÓN URGENTE DE RESOLVER

Transitar a un modelo de universidad emprendedora demanda poner en marcha con carácter de urgencia un cambio integral, instaurando el emprendimiento como un aspecto transversal en las tres funciones misionales de la universidad: enseñanza, investigación y proyección social, consolidándose como verdaderos centros de generación de ideas y de creación.

En el proceso de cambio propuesto es el aspecto formativo principalmente por el que debe empezarse, debiendo operar básicamente reformas que involucren la reorientación de los contenidos curriculares con enfoque hacia el desarrollo de la capacidad emprendedora de los estudiantes, convirtiendo el aula en un lugar y oportunidad para influir en la actitud emprendedora de los estudiantes de todos los programas académicos y brindándoles los espacios pertinentes para que se forjen como creadores y realizadores de proyectos teniendo en cuenta que el emprendedurismo se da en todos los ámbitos no estando necesariamente ligado a las carreras del área económico-empresarial o industrial..$^{13}$

\section{EL DESAFÍO QUE REPRESENTA PARA} LA UNIVERSIDAD NACIONAL MAYORDE SAN MARCOS LA INCURSIÓN EN LA INCUBACIÓN EMPRESARIAL

El potencial de conocimiento que existe en la Universidad Nacional Mayor de San Marcos (UNMSM) constituye un valioso capital para el desarrollo de incubadoras de empresas dentro de la Universidad, lo que se constituiría como un potencial intelectual que podría plasmarse en una aplicación real para la superación de la pobreza y la creación de riqueza.

En la Universidad Nacional Mayor de San Marcos se han realizado acciones importantes para fomentar el emprendedurismo universitario, como por ejemplo la suscripción de un convenio interinstitucional con la INICTEL-UNI en el presente año; así como también se han realizado algunos eventos organizados tanto por parte de los Vicerrectorados Académico y de Investigación como por parte de algunas Facultades de la UNMSM.

Así mismo hay algunos trabajos de investigación que se han ocupado de esta temática, como la tesis presentada el año anterior por dos docentes de la Facultad de Ingeniería de Sistemas, denominada: Modelo de comercio electrónico para incubadoras de empresas de base tecnológica aplicado a la UNMSM, exponiéndose un modelo de financiamiento para una incubadora de empresa de base tecnológica que se desarrollaría mediante comercio electrónico.

Todas estas iniciativas evidencian que sí hay interés por parte de los responsables del gobierno de la universidad de incorporar la dinámica del emprendimiento a la vida académica universitaria e institucional de la UNMSM. No obstante la realidad es que siguen siendo insuficientes porque aún no se emiten las políticas necesarias que traduzcan esa intención, que sí son captadas en seminarios y jornadas difundidas.

Mientras ello no suceda, en San Marcos todo lo que se ha mencionado quedarán

13 Becerra, Ana María y La Serna, Carlos. Documento de trabajo: Las competencias que demanda el mercado laboral de los profesionales del campo económico-empresarial en la actualidad. 
como hechos aislados pues es necesaria la creación de un programa de mayor trascendencia con estrategias y metas claramente definidas.

En este punto es pertinente mencionar los cinco ejes referidos por el Programa Universidad - Empresa para el desarrollo sostenible $^{14}$ para la implementación de un verdadero programa universidad-empresa, y que son: Actualización del currículo, financiamiento de la investigación, intercambio académico-empresarial, fomento de un nuevo modelo de pasantía y ejercicios profesionales aplicados y un programa de incubación empresarial, los cuales son verdaderos desafíos para el propósito planteado por la UNMSM.

\section{ASPECTOS DE INTERÉS COMÚN EN LA CONCRECIÓN DE UNA VINCULA- CIÓN CON LA EMPRESA}

Para la concreción de ese apoyo al desarrollo del país se hace necesaria la vinculación efectiva con la empresa y el gobierno, claro está que aunque la misión de cada uno es distinta también es claro que el marco común de colaboración supone una mutua conveniencia de satisfacer necesidades de ambas partes, pero por sobre todo la convergencia en la innovación y el desarrollo.

En este sentido, entre los aspectos que definen un interés común entre universidad y empresa cabe distinguir:

La coherencia y pertinencia de los programas académicos y los métodos educativos con las capacidades que la empresa requiere de los profesionales y técnicos. Este es un aspecto que interesa a ambas partes pues es un objetivo común buscar la satisfacción y aspiración de los jóvenes para labrarse un futuro personal y profesional satisfactorio.
Una previsión conjunta respecto a la posible evolución de las necesidades de la empresa como de los estudiantes. En un ejercicio de previsión y prospectiva en el que pueden requerirse contribuciones de otras disciplinas.

El intercambio de conocimientos y avances técnicos pues en determinados momentos alguna de las partes puede presentar alguna ventaja sobre la otra.

Una previsión de las implicancias de los avances científicos y tecnológicos que puedan darse en el futuro de las empresas y de las universidades, ideando juntos modos de enfrentar oportunamente dichos cambios previsibles.

El desarrollo conjunto de criterios y métodos para evaluar conocimientos y habilidades relevantes para los fines laborales y sociales.

La disminución del desconcierto que en los egresados produce el paso de la universidad al mundo laboral.

La facilitación de las necesidades de actualización y educación continua de los profesionales y técnicos de la empresa, así como de los académicos de las universidades, dado que lo que cada uno aporta es mutuamente complementario.

Resolución de problemáticas de la empresa con el conocimiento científico acumulado en las universidades y mediante la creatividad de los estudiantes ${ }^{15}$.

\section{ALGUNAS EXPERIENCIAS EXTRAN- JERAS DE INCUBACIÓN DE EMPRE- SAS EN UNIVERSIDADES}

Incubadora de Empresas del Instituto Tecnológico Superior de Monterrey-México:

14 Wehrtmann, I.S. \& M. Wesseler (eds). (2010). Universidades y empresas: generando potencial innovador: San José, Costa Rica. 15 Reséndiz Núñez, Daniel. La vinculación de universidades y empresas, un asunto de interés público y privado. 
A pesar de sus limitaciones presupuestales, México es un modelo de referencia en América Latina en cuanto a la incubación empresarial se refiere. Su primer programa de incubación de empresas se creó en 1990. El modelo mexicano de incubadoras ha tomado fuerza en el país, pues el Sistema Nacional de Incubación de Empresas está integrado por 500 incubadoras con presencia en 190 ciudades, según los registros de la organización México Emprende, de la Secretaría de Economía, de los cuales 258 son patrocinadas por universidades. Este país tiene asimilado el pensamiento de que el camino para una solidez de las Pymes -claves en la economía de cualquier país- está en la tarea de incubar profesionalmente las iniciativas emprendedoras de la población y particularmente de la juventud mexicana.

El Instituto Tecnológico Superior de Monterrey (ITESM) crea su Incubadora de Empresa en el 2009 con el objeto de ofrecer a sus alumnos, egresados y comunidad emprendedora un modelo de desarrollo de empresas que facilite su implantación, consolidación y mejora, así como apoyar a la formación de empresarios y empresas competitivas en el ámbito nacional e internacional con habilidades y actitudes que contribuyan al crecimiento y desarrollo social de su comunidad.

Su modelo de incubación ofrece dos modalidades de desarrollo: una modalidad física y una virtual. En la modalidad física brinda al emprendedor espacios físicos, infraestructura y servicios de apoyo para el desarrollo empresarial; en la modalidad virtual, ofrece al emprendedor servicios de apoyo para el desarrollo empresarial a través del portal emprendetec.com. Asimismo su modelo de incubación contempla tres etapas: preincubación (elaboración de plan de negocio y constitución legal de la empresa), incuba- ción (operación del negocio) y posincubación (consolidación y crecimiento del negocio). A lo largo de todo el proceso brinda el soporte de tutoría y asesoría especializada.

\section{Instituto Génesis de la PUC-Río - Brasil}

Es una unidad de la Pontificia Universidad Católica de Río de Janeiro (PUC-Río), creada con el objetivo de transferir el conocimiento de la universidad a la sociedad a través de la formación de empresarios y generación de empresas para una mejora de calidad de la vida de la región donde se insertan. Opera como un ambiente de innovación a la vez de apoyo y estímulo para el desarrollo de emprendedores. Recibe apoyo del Consorcio de Desarrollo, órgano consultivo formado por socios de las instituciones que contribuyeron al desarrollo y financiamiento de las acciones del instituto.

La incubadora del instituto se inauguró en julio de 1997. En su dinámica de operación actúa como canal de transferencia de conocimiento entre la universidad y la sociedad, apoyando empresas innovadoras en tecnologías de la información en las áreas de telecomunicaciones, educación a distancia, gestión del conocimiento, energía, entretenimiento, medio ambiente, deporte y medicina.

La incubadora tecnológica de esta universidad acompaña y apoya los proyectos desde la confección del prototipo hasta la formación de redes y consorcios empresariales. Durante el período de incubación las empresas usan los espacios físicos así como los servicios ofrecidos por las unidades operacionales. Entre otras bondades cabe mencionar la proximidad de los centros de investigación de la PUC-Río así como la posibilidad de desarrollar redes con los demás emprendedores e incentivos fiscales. 
El enfoque de la incubadora tecnológica de la PUC-RIO son los negocios que impliquen conocimiento multidisciplinar con uso intensivo de tecnologías de la información. En este sentido, sus convocatorias privilegian empresas que provean información de cualquier forma (verbal, sonora, impresa o en video), y en cualquier parte, con proyectos innovadores e intensivos en capital intelectual dentro de sus áreas de interés.

\section{Incubadora de Empresas Innovadoras de la Universidad Nacional de Luján - Ar- gentina}

La universidad Nacional de Luján desde 1995 mantiene un programa de incentivos para la creación de empresas innovadoras que se denomina "Programa Emprendedor" siendo su mayor enfoque crear un espacio para el proceso de transferencia del conocimiento científico tecnológico orientado al sector productivo, mediante el desarrollo de una cultura emprendedora que promueva la generación y crecimiento de nuevas empresas innovadoras.

Este programa considera la realización de sus tareas en todo el ámbito universitario. A su vez tiene propuesto un conjunto de actividades en diferentes áreas complementarias de trabajo que contribuyen al logro de sus objetivos. Estas actividades, cuyo número varía según las necesidades del programa, guardan relación directa con las actividades misionales de universidad: docencia, investigación, extensión y transferencia. Entre las principales actividades que contempla el Programa caben mencionar: Comunicación institucional, formación empresarial, Incubadora de Empresas Innovadoras (INCUEI), línea de investigación, gráfica esquema funcional.

La Incubadora de Empresas Innovadoras (INCUEI) ostenta instalaciones propias, en donde las empresas pueden acceder a mó- dulos de alojamientos individuales los que tienen el equipamiento básico con espacios de uso común, como sala de reuniones, secretaría, aula de capacitación y cafetería. Así mismo esta área presta servicios compartidos tales como: oficina, vigilancia, limpieza y mantenimiento.

\section{Incubadora de Empresas de Base Tecno- lógica de Antioquia - Colombia}

La Incubadora de Empresas de Base Tecnológica de Antioquia (IEBTA) se fundó en 1996 como organización mixta privada. Su creación auspiciada por más de 29 instituciones del sector productivo, académico, público y gremial, surge como respuesta a una necesidad latente de generación de espacios que motiven la renovación de la clase empresarial, así como la generación de la cultura emprendedora y marcar el inicio de la era del conocimiento en Antioquia.

Cumplidos el 2006 sus diez años en operación, esta incubadora se convirtió en un centro integral de servicios empresariales (CREA-ME), orientado a fortalecer y dinamizar la labor de empresas ya existentes, las ideas de negocio y los planes de desarrollo municipales.

Este centro integral opera como una agencia para la formulación, gerencia e implementación de empresas, programas, proyectos y estrategias de desarrollo productivo, competitivo y empresarial. Su operación está dividida en centros de servicios especializados, en el 2005 muestran los siguientes resultados: 39968 personas sensibilizadas en procesos de emprendimiento, 13532 personas formadas a través de cátedras virtuales, 1041 planes de negocios acompañados en su elaboración, 364 procesos empresariales con 1422 emprendedores, 113 inversionistas participaron en la primera rueda de inversiones, ventas de la comunidad empresarial 
por US\$ 12 millones. De acuerdo a lo informado en su web, la incubadora cuenta con 35 profesionales y con una extensa base de consultores nacionales e internacionales. En Colombia el equipo de la IEBTA ha participado en el diseño y puesta en operación de 13 nuevas incubadoras en todo el país y dos centros de emprendimiento empresarial.

En la actualidad es considerada una empresa altamente posicionada en el acompañamiento para elaboración y dirección estratégica de planes de negocio, lo que es evidente considerando los resultados de capitalización, alianzas estratégicas y de negocios de la mayoría de sus empresas y de su trabajo en consorcio con importantes consultoras de negocios.

\section{CONCLUSIONES:}

1. La incorporación de la cultura del emprendimiento al interior de la universidad debe concretarse desde un enfoque sistémico e integral, pues afecta las tres funciones misionales de la universidad: enseñanza, investigación y proyección social.

2. La incubación empresarial se encuentra aún en una fase inicial de desarrollo. Es importante que las universidades asuman un liderazgo en el tema de la incubación empresarial, forjando una nueva generación de líderes empresariales perseverantes y capaces de enfrentar riesgos.

3. Son pocas en la actualidad las instituciones universitarias que no hayan emprendido en el reto de la incubación empresarial, por lo que no hay razón para que San Marcos, la primera universidad pública del país, no incursione en un proyecto de esta naturaleza ya que aún hay un potencial mercado para gestionar emprendimientos.
4. Las condiciones actuales a nivel país se presentan más favorables para el desarrollo de la incubación de empresas que en el pasado, lo cual se refleja en una significativa cantidad de instituciones peruanas de diversa índole actuando en el tema.

5. Es importante para el movimiento de incubadoras peruanas su inserción en espacios y redes de incubadoras de la región, los cuales pueden permitir un mayor intercambio de información, así como establecimiento de diversas relaciones interinstitucionales.

\section{REFERENCIAS BIBLIOGRÁFICAS}

1. AWAD AUBAT, Gabriel. (2004). Diseño de un modelo de incubación de negocios utilizando herramientas de dinámica de sistemas. Tesis.

2. CONAM-CAF-GEF.(2005). "Identificar acciones específicas a ser instrumentadas para el fortaleciento de las actividades de apoyo al biocomercio en las Incubadoras de Empresas". Recuperado de sitio web:

http://www.caf.com/attach/9/default/ Peru-8Accionesparaincubadorasde empresas.pdf

3. DELICIO FABIÁN, ANDRÉS(2006). Estrategias para el fomento del emprendedurismo en la Facultad de Ciencias Económicas y Sociales de la Universidad Nacional del Mar del Plata. Año 12, No 26.

4. RESÉNDIZ NÚÑEZ, DANIEL. (1998). "La vinculación de universidades y empresas: un interés público y privado". Recuperado de sitio web: http://www. anuies.mx/servicios/p_anuies/publicaciones/revsup/res106/txt5.htm

5. VELA VELÁSQUEZ, Julio César. (2011) Modelo para la creación de in- 
cubadoras de empresas en la realidad peruana. Tesis. Recuperado de sitio web: http://tesis.pucp.edu.pe/repositorio/ bitstream/handle/123456789/914/ VELA_VELASQUEZ_JULIO_INCUBADORAS_REALIDAD_PERUANA. pdf ? sequence $=1$
6. WERHTMANN, Ingo S. \& WESSELER, Matthias. (2010). "Universidades y empresas: generando potencial innovador. San José de Costa Rica”. Recuperado de sitio web: http://innovacion.org.gt/ sites/default/files/Universidades\%20 y\%20Empresas.pdf 